\title{
GÉNESIS DE LA NOCIÓN DE SUSTANCIA ESPIRITUAL EN LA FILOSOFÍA DE GEORGE BERKELEY. I
}

\author{
José A. RoBles
}

Instituto de INVESTigaciones Filosóficas Universidad Nacional Autónoma de México

0. Introducción. En el presente escrito,* que aún tiene el carácter de un cúmulo de notas con - según espero cierta unidad sobre el tema que señala el título, surge de una ponencia - que fue, también, un conjunto de notas, aún menos elaboradas que el texto actual- que presenté en el II Congreso Nacional de Filosofía (1983), organizado por la Asociación Filosófica de México. La razón que tuve para no dar el texto original para su publicación en las memorias del Congreso, fue el carácter muy tentativo y desordenado -en parte- que consideraba que tenía dicho escrito. En esta nueva redacción he intentado eliminar algunas de esas deficiencias, pero manteniendo aún la idea de que el texto ha de verse como un conjunto organizado de notas de un trabajo en proceso sobre el tema señalado.

0.1. Motivación. La motivación del presente trabajo la origina la asimetría que hay en la filosofía de Berkeley en las posiciones divergentes que mantiene con respecto, por una parte, a la sustancia material y, por otra, con respecto a la sustancia espiritual. Como es bien sabido, Berkeley rechaza la sustancia material (lockeana) por considerar que la misma (en caso de que hubiera algo así) nada añade a nuestra capacidad de conocer (y entender) el mundo externo (perceptual); la conclusión de Berkeley acerca de esto no es tan sólo la de señalar que la sustancia material (lockeana) es inútil sino, peor aún, que es imposible. Sin embargo, su tratamiento de la sustancia espiritual es el opuesto: hay sustancias espirituales que son los sujetos que conocen y tienen actividades volitivas. La pregunta que surge de inmediato es la relativa a esta asi-

- En el Seminario de Investigadores del IIF lel una versión anterior de este escrito en la que, entre otras diferencias menores, no presentaba el texto de Hume. Agradezco a Enrique Villanueva su participación como replicante principal en ese Seminario, así como las intervenciones de Luis Aguilar Villanueva, Mauricio Beuchot, Alejandro Herrera, Mark Platts y los comentarios que, fuera de la sesión del Seminario, me hiciera el Dr. Manuel Cabrera. En la parte II de este escrito espero dar cuenta y razón adecuadas de las útiles sugerencias que me proporcionaron. 
metría y responder esta pregunta es la meta que persigo en esta investigación en proceso.

Al lector del presente texto le será evidente que no respondo la pregunta, pero considero que algunas de las observaciones que han surgido en mi proceso de búsqueda pueden serle de interés. Lo que más ha atraído mi atención es el estudio del tema en los Philosophical Commentaries $(P C)$ berkeleyanos, donde es posible ver la gestación de los temas centrales de la filosofía de Berkeley y donde considero que puedo encontrar una respuesta a mi perplejidad.

Lo que hago en el texto principal es, primeramente, recordarle al lector, mediante un esbozo muy comprimido, las propuestas lockeanas básicas sobre el tema de la sustancia material y las críticas que Berkeley lanza en su contra. De inmediato tomo las notas de Berkeley en los PC e intento seguir el curso de su pensamiento acerca del tema de la sustancia espiritual. Lo que me parece importante señalar es que, al iniciar sus investigaciones sobre el tema, Berkeley está fuertemente influido por una tesis semántica lockeana (a toda expresión significativa ha de corresponderle una idea; cfr. Essay III, ii, 1 y 2, p. ej.) y, debido a esto, llega a proponer una tesis de lo que sea el espíritu (el alma; Berkeley emplea los términos 'soul', 'spirit') que en nada difiere de la que posteriormente propusiera Hume: no hay una sustancia espiritual individual, además de la conjunción (haz, cúmulo; Berkeley usa la palabrạ 'congeries' a diferencia del 'bundle' humeano pero, fuera de una distinción verbal así, las doctrinas son similares) de perceptos que es el que constituye a un individuo. La conclusión la alcanza Berkeley por haberse convencido de que las palabras que aluden a una entidad activa (como lo sería el alma en caso de haber algo así) no pueden aludir a (o tener un correlato con) ideas -pues éstas son pasivas, inertes- y, por esto, tales palabras carecerian de significado. Berkeley pronto se da cuenta, sin embargo, que la tesis semántica que lo ha llevado a una conclusión ontológica semejante no puede ser correcta, pues tendría que concluir, conforme a ella, que una idea -en el sentido berkeleyano del término- puede tener - percibir, ser activa - otras ideas; esto es, un ser humano, p. ej., en tanto que se le considere tan sólo como el conjunto de todas sus manifestaciones sensibles -figura, tamaño, color, etc. - y como tal capaz de ser considerado en términos ideistas, podría percibir y actuar de otras formas con respecto a otras ideas. Berkeley considera, entonces, que la tesis semántica debe modificarse de tal manera que las expresiones que aluden a entes activos __Dios' sería un ajemplo central de esto- no sean asignificativas (a pesar de que no sea posible representar tales entes mediante ideas). Este cambio semántico tiene gran importancia en la visión filosófica de Berkeley pues el mismo 
le permitirá tratar con mayor libertad no tan sólo temas relacionados con aspectos de filosofía moral, sino incluso problemas relacionados con la ciencia de su tiempo, y adoptar en la matemática, p. ej. al considerar la aritmética y el álgebra, una posición netamente nominalista, o bien, en general, tanto en la matemática (en geometría de manera expresa) como en física llegar a postulaciones pragmático-instrumentalistas. ${ }^{1}$ Finalmente, aún queda por aclarar la necesidad que sintiera Berkeley de abandonar la posición humeana (avant la lettre) a la que he aludido y postular una sustancia espiritual. Dar cuenta y razón de esto, sin embargo, es la finalidad de mi trabajo en su totalidad y, en la etapa en que éste se encuentra, todavía no tengo ninguna visión clara de este asunto.

0.2. Los Philosophical Commentaries. El texto de Berkeley que utilizo para mi trabajo es el conocido con el título de Philosophical Commentaries que A. A. Luce editara en 1944. Los $P C$ son un par de cuadernos de notas $(A$ y $B$ ) que Berkeley redactó entre los años de 1707-1708 (inicia su redacción a la edad de 22 años), en los que se muestra el proceso de gestación, crecimiento y maduración de las ideas centrales de Berkeley acerca de los más diversos temas. Alexander Campbell Fraser descubre los $P C$ y los publica en 1871 —en su edición de las obras de Berkeley, vol. IV en donde figuran con el título de Commonplace Book of Ocasional Methaphysical Thoughts-, tan sólo que - -según lo demuestra Luce en su edición de 1944; cfr., bibliografía- fueron publicados en el orden equivocado y esto impidió que, desde un principio, fueran adecuadamente evaluados con respecto a la importancia que tienen para adquirir una comprensión mejor de la filosofía de su autor. Luce los edita en el orden $B, A$ y al hacer esto puede verse el desarrollo del pensamiento berkeleyano hasta la etapa inmdiatamente anterior a la publicación de dos de sus obras centrales: Theory of Vision y Principles of Human Knowledge, Part I (obras publicadas, respectivamente, en 1709 y 1710). De los $P C$ estoy preparando una edición (traducción, introducción y notas) que considero vendrá a llenar una laguna, en nuestra lengua, con respecto a las obras del filósofo irlandés y ayudará a obtener una mejor comprensión de las tesis de este pensador original y profundo.

Precisamente es en los $P C$ donde Berkeley registró los pensamientos que gradualmente se configuran en sus tesis filosóficas conocidas; mi interés en el presente trabajo, según lo he señalado, es considerar el proceso de formación de una de tales tesis y el único lugar donde puede obtenerse un conocimiento de primera mano de tal proceso es, justa-

1 Estos temas los he tratado in extenso en mi libro en prensa, Las ideas matemá. ticas de George Berkeley, Obispo de Cloyne. 
mente, en los $P C$. Esto señala, de manera mínima, la importancia que puede tener el estudio de los mismos.

1. Locke, Berkeley y la sustancia material. La posición filosófica de Berkeley con respecto a nuestro conocimiento del mundo exterior se ha prestado a muy diversas interpretaciones que, en un número considerable de casos, han distorsionado notablemente su pensamiento. La situación ha cambiado en los últimos tiempos y un intérprete como Pitcher $-c f r$., bibliografía - nos ofrece, en su trabajo, un pensamiento berkeleyano rico, variado y fecundo; quien desee conocer en detalle alguna de las facetas de la argumentación berkeleyana podrá asomarse con provecho a sus páginas. Lo que presentaré a continuación es sólo un bosquejo de las propuestas tanto de Locke como de Berkeley con relación a la sustancia material, esperando que la distorsión que produzca no sea excesiva, gracias a estas palabras de advertencia.

Una posición que es bien conocida por casi cualquier estudiante de los primeros años de filosofía es la que mantiene John Locke, el ilustre antecesor de Berkeley, acerca del conocimiento del mundo exterior. La tesis de Locke que aquí me interesa presentar, en un apresurado esquema, puede formularse en los siguientes términos: hay un mundo exterior. Este supuesto, según lo he discutido con Carmen Silva, parece ser algo que naturalmente se impone a algunos pensadores del siglo xvir: los avances notables que en ese siglo empieza a lograr la ciencia natural parece que impedirían pensar de otra manera. La matemática de la época estaba sufriendo una evolución y un progreso notables urgida por los prolemas que se les presentaban a los investigadores de la época y a sus antecesores inmediatos: Kepler en busca de las orbitas planetarias; Fermat, Descartes, Huyghens, Boyle, etc., enfrentados a problemas de curvatura de lentes, problemas logísticos, péndulos, curvas isócronas, problemas de isoperimetría, etc., y, finalmente, Newton, calculando la atracción gravitatoria y preocupado por otros mil problemas científicos (y teológicos), siguiendo los pasos de sus notables antecesores, como Barrow (su maestro en Cambridge), Wallis, Cavalieri, etc.

Pero volvamos a Locke. Este filósofo, según lo señalé, mantiene como supuesto ontológico básico que hay un mundo externo. Ahora bien, su forma de explicar cómo es que entramos en contacto epistémico con este mundo radica en apelar a las teorias atomistas y mecanicistas de la época y en establecer una relación causal entre el mundo externo y nuestros órganos de sensación: los objetos del mundo exterior afectan nuestros organos sensoriales por la emisión de pequeños corpúsculos que al chocar con las terminaciones nerviosas de nuestros órganos sensoriales, los activan y el resultado de esta activación es que, en nosotros, se producen 
ideas de los objetos externos. El sujeto cognoscente, pues, tiene contacto epistémico con los objetos externos a través (por medio) de las ideas que en él producen tales objetos. Su conocimiento de éstos, entonces, es indirecto (mediato), por medio de las ideas, de las que tiene un conocimiento directo (inmediato).

Un rasgo más de la posición lockeana que me interesa destacar, es el siguiente: aun cuando el sujeto conozca directamente sólo sus ideas, éstas le proporcionan, sin embargo, un conocimiento verdadero de algunas de las características o cualidades de los objetos productores de ideas, tal como las mismas (las cualidades) se encuentran en ellos: Locke distingue entre ideas de cualidades primarias e ideas de cualidades secundarias de los objetos. Las ideas de las cualidades primarias representan las cualidades primarias (figura, reposo-movimiento, dureza, ...) tal como éstas se encuentran en el objeto; en cambio, las ideas de las cualidades secundarias representan sólo potencias en el objeto para producir tales ideas, sin que haya cualidades asi en el objeto. Una mínima observación acerca de las cualidades primarias: éstas eran las que la ciencia podía tratar sin problemas; la geometría podía tratar, de manera directa y precisa (según la concepción de la época) con la forma de una figura plana, con el volumen de un cuerpo; los matemáticos podían determinar centros de gravedad, calcular el área de superficies bordeadas por curvas $\rho$ bien determinar la velocidad instantánea de cuerpos en movimiento, etc. Esto no era asi con los colores, olores, sabores, etc., por lo que estas propiedades, en tanto que ideas, no tenían un correlato exacto en el cuerpo que las producía. La visión del mundo que Locke nos ofrece mediante su teoría epistémica parece que corresponde de manera bastante precisa con nuestra visión del mundo de sentido común. Sin embargo, hay algo en esta teoría que impide que la misma sea tan diáfana y distinta como estaríamos tentados a creer. Locke mismo señala la dificultad al decirnos que de la causa misma de nuestras ideas no podemos tener un conocimiento directo. Detrás del velo de las ideas se encuentra la causa de éstas, pero el velo no lo podemos descorrer. Locke habla de un sustrato material incognoscible que es el generador de nuestro conocimiento. Así, nos dice:

Pues en ambos casos [tanto en el caso de la sustancia material como en el de la espiritual] nuestra idea de sustancia es igualmente oscura: no es sino un no sé qué supuesto como soporte de esas ideas que denominamos accidente (Essay II, xxiii, 15).

Berkeley descubre en esto el origen del escepticismo y de grandes errores en la posición materialista de otros filósofos. Lo que Berkeley encuentra criticable en la postura de Locke, filósofo al que él cierta- 
mente admira y respeta, es que tras haber dado una descripción, que él (Berkeley) considera lúcida y adecuada, acerca de nuestro conocimiento del mundo externo, llegue a postular un sustrato incognoscible como generador de nuestras ideas. Pero, señalará Berkeley, ¿tenemos necesidad de un sustrato así para dar cuenta de nuestro conocimiento del mundo? Por otra parte, Berkeley encuentra problemas en la posición de Locke cuando éste presenta su génesis causal de las ideas: jpodemos afirmar que algo desconocido y, además, incognoscible es causa de nuestras ideas? ¿Cómo podemos fundar esta afirmación de relación causal? El resultado al que Berkeley llega, luego de analizar la posición de Locke, es que la propuesta de éste, con respecto a nuestno conocimiento del mundo externo, es correcta en lo tocante a que sólo tenemos un conocimiento directo de ideas; que lo que Locke añade, acerca de la relación causal con un no sé qué incognoscible, es inútil e incluso contradictorio en tanto que no podemos afirmar nada acerca de lo que desconocemos y que, incluso, es incognoscible, por lo que si eliminamos el sustrato material del cuadro que Locke nos da de nuestro conocimiento del mundo externo, no estamos eliminando algo que sea real, sino sólo palabras que nos confunden, haciéndonos pensar que debe haber algo más detrás del 'velo' de las ideas. Estas ideas, de acuerdo con Berkeley, no son velo alguno, sino que nos presentan el mundo perceptual tal como éste es.

La crítica de Berkeley, claro está, se encuentra más matizada en sus escritos y antes de llegar al rechazo de la idea sustancialista de Locke señala que los argumentos lockeanos para mostrar que las ideas de cualidades secundarias no nos presentan algo en el objeto, pueden usarse igualmente para mostrar lo mismo acerca de las primarias. El dictamen de Berkeley es que ambos tipos de ideas de cualidades se encuentran a la par y que no es mediante los argumentos de Locke como vamos a poder darles primacía a unas con respecto a las otras.

Sin embargo, no es el detalle de los argumentos berkeleyanos lo que aquí me interesa considerar, sino la agudeza lógica de su crítica a la posición que guarda, dentro del esquema epistémico de Locke, la idea de sustancia, asi como su crítica a esta misma idea. Si es correcta la lectura que hace Berkeley de la tesis de Locke, entonces también lo es su conclusión en el sentido de que al eliminar la sustancia material lockeana no eliminamos nada en el mundo que creamos que en él existe conforme a nuestra visión de sentido común del mismo. En los $P C$ Berkeley nos dice:

M No quito sustancias. No se me debe acusar de descartar la sustancia fuera del mundo razonable. Sólo rechazo el sentido filosófico (que, en efecto, no es sentido) de la palabra sustancia. Pregúntesele 
a un hombre nunca $\dagger$ expuesto a la jerga de ellos qué es lo que quiere decir con sustancia corpórea, o la sustancia de un cuerpo. Él responderá Masa, Solidez y cualidades sensoriales similares. Estas las retengo. el nec quid nec quantum nec quale Filosófico del cual no tengo idea lo descarto. si de un hombre se puede decir que descarta aquello que nunca tuvo ser alguno nunca fue ni siquiera imaginado concebido. ${ }^{2}$

Que lo último que nos señala Berkeley es así se sigue debido a que, según su lectura, la propuesta de Locke es contradictoria, por lo que una contradicción no puede ser una descripción de algo que realmente exista (ni de cosa alguna, en general). Esto ilustra, también, por qué no demuestra nada la supuesta "refutación" de las doctrinas de Berkeley que "expresara" el Dr. Johnson pateando una piedra: ${ }^{3}$ Berkeley

2 Todas las citas de Berkeley que figuran en este escrito son de los $P C$; la presente es $P C$ 517, lo que señala el número que la nota tiene conforme a la edición definitiva de Luce. Las demás citas las anoto de manera similar en su lugar de aparición. Con respecto a la extraña puntuación, así como al uso de mayúsculas en lugares inesperados $\mathrm{e}$ incluso la redacción que resulta ambigua en muchos casos en las anotaciones de los $P C$ y el nombre "Malbranch" en lugar de "Malebranche" que figura en alguna nota, señalo que se debe a que he intentado apegarme, lo más posible, al texto de Berkeley, tal como lo estableciera Luce en su edición de esta obra.

Una observación relacionada con las observaciones marginales, que figuran en las notas de los $P C$ es la siguiente: Berkeley empleó diferentes signos para señalar el tema que trataba en cada una de sus notas. Daré aquí una breve lista de los que figuran en las citas que presento, asi como su significado:

el signo " $t$ ", que es el que con mayor frecuencia figura en las citas, es el signo que empleó Berkeley para señalar, en la mayoría de los casos, que no estaba de acuerdo con lo expresado en la nota; es, así, un signo que señala una modificación doctrinal en su pensamiento.

"X" "Matemática; "M" Materia; "E" Existencia;

"S" (Spirit-Soul) Esplritu-Alma; "G" (God-Dios;

"I" Introducción (a los Principios del conocimiento humano).

Un par de observaciones más, acerca de las notas citadas, es el siguiente: algunas de las notas de los PC comienzan con "Pr:", léase esto como "Preguntas" - en el original inglés Berkeley pone "Qu:", de "Question", "Quaere” o "Quaestic"-. Finalmente, algunas notas, en la enumeración de Luce, tienen inmediatamente después del número de nota una letra "a" - p. ej., 194a-; esto señala que la nota en cuestión es un comentario, ampliación o enmienda de la nota con el mismo número. Así, 194a formula una pregunta — duda - que le surge a Berkeley al considerar la nota 194 -cfr., p. 13.

Quien desee mayores datos sobre el texto de los $P C$ puede consultar la bien documentada introducción de Luce en [1], pp. xiii-xli.

3 La famosa 'refutación' de Johnson aparece en The Life of Samuel Johnson (1785) de James Boswell, donde su autor narra que él y Johnson se diriglan a Harwich; en el viaje se detuvieron en Cochester y a la mañana siguiente de su llegada a esa localidad, visitaron la iglesia. Boswell escribe, "al salir de la iglesia, nos detuvimos por un rato a hablar de los ingeniosos sofismas del Obispo Berkeley para demostrar la no existencia de la materia y que todo en el universo es tan sólo ideal. Yo hice la observación de que aun cuando estábamos convencidos de la no verdad de su doctrina, era imposible refutarla. Nunca olvidaré la vivacidad (alacrity) con la que res- 
no eliminó, del mundo, las ideas ni nuestras sensaciones; lo único que eliminó fue una propuesta pseudoexplicativa de Locke, que éste ofrecía en términos de la supuesta idea de "sustancia material". Lo que Berkeley expresó en su crítica a Locke puede formularse en los siguientes términos: la propuesta de Locke acerca de nuestro conocimiento del mundo exterior será correcta si eliminamos de ella ciertos aspectos de la misma que son contradictorios (y que, por tanto, nada describen), lo que hace que obtengamos una imagen oscura e incorrecta de cómo es el mundo exterior. Lo que Berkeley propone, entonces, es eliminar las contradicciones de la posición de Locke y, así, hacerla consistente. Si lo que digo es correcto, una crítica a la propuesta de Berkeley no podrá hacerse aceptando, sin más, una posición del tipo de la de Locke y lanzando, desde ella, el cargo de que Berkeley nos ha privado de algo esencial en nuestra visión del mundo. Un crítico lockeano de Berkeley tendrá que mostrar, o bien $a$ ) que la posición de Locke no es inconsistente y que, por esto, falla la crítica de Berkeley, o bien b) que es posible levantar el velo de las ideas y tener un "conocimiento" de algún tipo (no necesariamente perceptual) del sustrato lockeano.

Concluyo esta sección haciendo un breve resumen de mis observaciones:

i) tanto Locke como Berkeley aceptan que sólo puede tenerse un conocimiento directo, inmediato, de ideas;

ii) Locke postula una génesis causal de nuestras ideas y, para explicarla, apela a las nociones de movimiento y choque de pequeñas partículas, que provienen del objeto, con las terminaciones nerviosas de nuestros órganos sensoriales (no explica, sin embargo, cómo por estos choques se producen las ideas);

iii) en el apartado anterior hablé del objeto productor de las ideas, pero esto es algo que queda fuera del mundo de aquéllas: el objeto produce las ideas, pero no tenemos una idea de él; las ideas que tenemos corresponden a cualidades del objeto, algunas de manera precisa (las ideas de cualidades primarias), otras de manera imprecisa (ideas de cualidades secundarias).

iv) Berkeley encuentra problemática la postura de Locke en tanto que éste deja fuera del campo de las ideas al objeto mismo que según Locke las produce;

v) la debilidad que Berkeley encuentra en esto es que, entonces, se desconoce (y es incognoscible) la causa de las ideas, por lo que

vi) ésta no puede jugar papel alguno en la explicación de la génesis de nuestras ideas. Conclusión,

pondió Johnson, estrellando su pie con enorme fuerza contra una gran piedra, hasta rebotarlo de ella, 'Yo la refuto asf'" ". 
vii) eliminar el sustrato material lockeano de nuestra epistemología, no elimina nada, pues la postulación misma de tal sustrato no es inteligible.

Ciertamente Berkeley no es muy claro en el detalle de sus críticas, según lo han mostrado algunos de sus comentadores; y, ciertamente, también es posible leer a Locke de alguna manera diferente a como lo hiciera Berkeley. Sin embargo, en mi conclusión general, en este apartado, me uno a lo que observa Pitcher. Dado que tanto Berkeley como Locke conceden que, en la percepción, el perceptor conoce directamente (inmediatamente) sólo sus ideas, Pitcher señala que: *

Berkeley impugna a Locke precisamente en los aspectos correctos.

He sugerido que un lockeano -continúa Pitcher- podría ser capaz de responder estos cuestionamientos, pero debe responderlos y hacerlo adecuadamente, aun si, en efecto, puede hacerse, y esto será un proyecto largo y de difícil realización.

2. Sustancia espiritual. Luego de haber considerado la crítica de Berkeley a la idea lockeana de sustancia material, uno estaría tentado a pensar que Berkeley adoptaría una posición similar con respecto a la idea de sustancia espiritual. Ciertamente uno no sufriría una decepción si leyese las anotaciones en $\operatorname{los} P C$, desde la primera hasta la primera mitad de las notas 600 . En esta etapa temprana del desarrollo del pensamiento berkeleyano uno encuentra una posición que difícilmente se distingue de la posterior de Hume, si es que acaso hay diferencia alguna entre ellas; en anotaciones posteriores se empieza a perfilar la tesis oficial, o publicada, de Berkeley, pero en el desarrollo de la redacción de los $P C$ pueden observarse matices y detalles que luego no son tan claros o desaparecen completamente en la obra publicada. Aquí presentaré un breve bosquejo de la propuesta berkeleyana anticipatoria de Hume, hasta que hacen su aparición algunas dudas y problemas —en los $P C$ que harán que Berkeley modifique su posición.

La primera anotación que me parece importante destacar aquí es:

I. S. El discurso es más metafórico de lo que imaginamos pues expresa en su mayor parte cosas insensibles y sus modos circunstancias etc. mediante palabras tomadas de las cosas sensibles. la razón es clara. Por tanto Errores Múltiples (PC 176).

y luego añade un comentario,

S El gran Error es que creemos tener Ideas de las Operaciones de 4 En [4], p. 151. 
nuestras Mentes. ciertamente este ropaje Metafórico es un argumento que no tenemos ( $P C$ 176a).

Lo que me interesa destacar de estas notas de Berkeley es, por una parte, a) la relación entre el lenguaje y las cosas insensibles y $b$ ) su negación de que hay ideas de las operaciones de la mente. La importancia que tienen estas dos observaciones es la siguiente: en la época del desarrollo filosófico de Berkeley que aquí considero, él sostuvo una posición semántica extrema en el sentido de mantener que cualquier expresión, para que sea significativa, debe representar alguna idea. Así, p. ej., en $P C 312$ señala "4 Principios mediante los cuales responder objeciones..." y como su principio 3, adopta una posición lockeana:

3. Todo el lenguaje y el conocimiento es acerca de ideas, las palabras no representan nada más;

en $P C$ 354, hablando de infinitesimales, nos dice:

$\mathrm{X}$ Axioma. Ningún razonamiento acerca de cosas de las que no tenemos idea alguna. Por tanto, ningún razonamiento acerca de Infinitesimales;

en $P C 356$ subraya la doctrina:

+ Axioma. Ninguna palabra ha de usarse sin una idea

y en $P C 378$ afirma tajante:

+1 . Todas las palabras significantes representan Ideas

(véanse, además, $P C 421,422$-estas anotaciones repiten $P C 354$ y 356 , respectivamente-, 494, 495 -en estas anotaciones empiezan a colarse algunas dudas sobre la tesis-, 522, 595, etc.).

Así pues, en esta etapa de su desarrollo filosófico, Berkeley no puede aceptar que haya palabras (significativas) sin alguna idea que les corresponda y, por otra parte, según leemos en 176a, no tenemos ideas de las operaciones de la mente, así que no podemos hablar de ellas; por último, teniendo en cuenta la tesis lockeana, expresada en $P C 312,3$, no podemos conocer las operaciones de la mente, pues todo nuestro conocimiento es de ideas. Hasta aquí las cosas, una de las primeras posiciones de Berkeley con respecto a las operaciones de la mente es agnóstica; pero si avanzamos un poco más en la lectura de los $P C$, nos encontra- 
mos con que en $P C 363$ y 363 a se anuncia el principio inmaterialista que finalmente formula en $P C$ 377:

\section{M. ${ }^{1}$ Una idea no puede existir no percibida}

y en $P C 378$ presenta una larga demostración del principio indicado de inmediato, en 378a, la necesidad de abreviarla y, por último, en $P C$ 379 y 380 , da un resumen de la misma. Entonces, con todos los elementos que hasta aquí he presentado es posible concluir, de manera definitiva, que Berkeley, hasta este momento de la redacción de los $P C$, debe mantener que no tienen sentido las palabras "operaciones de la mente". El argumento para llegar a esta conclusión puede resumirse en los siguientes pasos:

1. Todo nuestro conocimiento es de ideas ( $P C$ 313, 3)

2. De las operaciones de la mente no tenemos ideas (PC 176a)

$\therefore 3$. De las operaciones de la mente no tenemos conocimiento

4. Todas las palabras significantes representan ideas ( $P C$ 378)

$\therefore 5$. "Operaciones de la mente" no son palabras significativas.

La conclusión 3 surge claramente de 1 y 2, lo que representa una posición agnóstica con repecto a las operaciones de la mente; la conclusión 5 se deriva de 2 y 4 y esto impide que podamos hacer alúsión a alguna cosa tal como 'operaciones de la mente'. La pregunta que a uno se le ocurre formular de inmediato es si estaría Berkeley dispuesto a aceptar que hay cosas inefables; esto es, ces su propuesta, a la vez de semántica, una propuesta que conlleva tintes ontológicos o hay lugar para suponer que la realidad excede los límites del lenguaje, tal como Berkeley lo interpretaba en estos pasajes de los $P C$ ? Hay lugares en los $P C$ que dan pie para pensar que Berkeley deseaba acotar tan sólo límites expresivos mediante su tesis acerca del lenguaje y que la misma no acotaba ni límites epistémicos -a pesar de lo que hemos podido leer en contra de esto anteriormente- ni ontológicos. Por ejemplo, en $P C$ 178 , nos dice:

+ La imposibilidad de definir o de discurrir claramente acerca de la mayoría de las cosas procede de la falta y la escasez del lenguaje, tanto, quizás, como de la oscuridad y confusión del Pensamiento. Por tanto iyo puedo clara y plenamente entender la extensión de mi propia Alma, etc. y no ser capaz de definirlas!

Y más adelante dice: 
Conocemos muchas cosas que no podemos expresar por falta de palabras. Conforme a este Principio son descubribles grandes cosas, por no haberlo considerado diversos hombres han caído en varios errores al intentar formular sus conocimientos mediante sonidos y al fracasar en esto pensaron que éste era un defecto de su conocimiento cuando en verdad lo era de su lenguaje ( $P C$ 223).

Y siete anotaciones más adelante leemos:

S Absurdo que los hombres hayan de conocer el alma mediante idea por ser las ideas inertes, no pensantes, Por tanto refutado Malbranch ( $P C$ 230). i.

Estas tres últimas anotaciones son anteriores, ciertamente, a $P C$ 378; sin embargo, en $P C$ 429, señala:

E Existencia es percipi o percipere $†$...

y el comentario inmediato añade:

† o velle i:e. agere. (PC 429a).

Lo que estas anotaciones muestran, según creo, son dos cosas: i) Berkeley no limita su ontología, ni siquiera en esta primera época de su desarrollo filosófico, a lo expresable, y ii) en su pensamiento existe una gran tensión interna con respecto a su tesis semántica explícita y a lo que expresa en otras anotaciones. Esta tensión llegará a desaparecer una vez que se convenza de que no puede limitar la significatividad de su lenguaje a la representación ideista, pero esto saldrá más adelante con algún detalle. Por el momento, la posición de Berkeley es la ideista que he formulado y la conclusión que considero que se sigue de ella es la de la carencia de significado de ciertas expresiones que no corresponden con ideas. Por otra parte, creo que también es posible concluir que Berkeley no restringe su ontología a ideas, pero cualquier otra cosa que haya, además de éstas, es - hasta ahora - inefable.

Aún queda un problema por resolver: ¿cuál es la caracterización positiva que Berkeley puede dar de la mente en esta primera etapa de su desarrollo filosófico? Paso ahora a tratar este tema.

La preocupación por temas relacionados con la sustancia espiritual hace su aparición en anotaciones tempranas de $\operatorname{los} P C$, aun cuando no de manera directa. La preocupación más temprana de Berkeley se centra en lo que se ha dado en llamar "la conciencia inmanente del tiempo"; así, en $P C$ 14, dice: 
+ La eternidad es sólo una sucesión de innumerables ideas. por tanto fácilmente se concibe la inmortalidad del Alma. o, más bien, la inmortalidad de la persona, al no ser necesaria la del alma hasta donde podemos ver.

La persona comienza a jugar un papel destacado en su pensamiento. En $P C$ 24 dice:

+ Nada existe propiamente sino las personas, esto es cosas conscientes, todas las otras cosas no son tanto existencias como maneras de la existencia de las personas.

$\mathrm{Y}$, en la siguiente anotación, señala:

+ Pr: acerca del Alma o más bien de la persona si no es completamente conocida ( $P C$ 25).

En esta primera fase de su pensamiento, Berkeley habla tanto de alma como de persona, sin establecer una distinción clara entre estas dos entidades. Más adelante, en PC 713, abandona la expresión 'persona' pues, según nos dice:

S Lo Concreto de la Voluntad y el entendimiento debo denominarlo Mente, no persona, para evitar ofensas, pues no hay sino una volición reconocida que es Dios. Mem: Cuidadosamente omitir el Definir Persona o mencionarla mucho.

Lo que hace que Berkeley abandone el término es su temor de entrar en conflictos eclesiásticos por el uso Trinitario de "Persona" y así, en sus obras publicadas, no emplea la expresión.

Volviendo al tema de la posición de Berkeley con respecto al tema de la sustancia espiritual, vale la pena señalar aquí que hasta el punto al que hemos llegado, $P C$ 25, aún no se han puesto en reación alma e ideas. Por otra parte, en esta etapa de su pensamiento, Berkeley hace que todo lo existente sea, tan sólo, "cosas conscientes"; más adelante hará importantes distinciones. La tesis de $P C 24$ la precisa en $P C$ 52:

+ Los cuerpos etc. existen aun cuando no son percibidos pues son potencias en el Ser activo.

Algunas notas antes, sin embargo, había surgido ya una pregunta tentativa en el sentido de precisar lo que sea el alma: 
+ Pr: si el ser no podría ser la sustancia del alma, o (de otra manera así) si el ser añadido a las facultades $[$ [no] completa la esencia real y la definición adecuada del alma? ( $P C$ 44).

Entreveradas con estas anotaciones hay otras más en las que Berkeley considera problemas matemáticos de la época o bien problemas perceptuales con respecto a un ciego de nacimiento que adquiere la visión, etc. La consideración de estos últimos problemas le va permitiendo formular primero y luego precisar su tesis básica, esse est percipi, que aún no hace su aparición en los $P C$. Hasta que llegamos a $P C 154$, donde hay una propuesta más directa acerca de lo que sea el alma:

+ Por Alma sólo se quiere decir una idea Compleja compuesta de existencia, que actúa volitivamente (willing) y percepción en un sentido amplio, por tanto es conocida y puede definirse.

Esta primera propuesta positiva hace del alma una idea (compuesta) en cuya composición intervienen (¿una idea de) existencia (?), un elemento activo (¿idea, también?) y percepción. Esta primera caracterización positiva de "alma" que buscábamos resulta ser de gran oscuridad y, por otra parte, recordando lo que Berkeley nos dice en 176a, de las operaciones de la mente no tenemos ideas, por lo que Berkeley rechazará casi de inmediato esta primera propuesta de caracterizar el alma. Sin embargo, en esta propuesta cabe notar que entran en contacto alma e ideas, siendo la primera un compuesto de las últimas.

Una propuesta más promisoria aparece en $P C$ 194, donde la preocupación es, una vez más, la identidad personal:

+ Conforme a mi doctrina la identidad de las sustancias finitas debe consistir en algo más que existencia continua o relación a determinados tiempo y lugar de comenzar a existir, la existencia de nuestros pensamientos (los que combinándose hacen todas las sustancias) se interrumpe frecuentemente y ellos tienen diversos comienzos y terminaciones.

De inmediato, se pregunta:

$S$ Pr: Si la identidad de la Persona no consiste en la Voluntad (PC 194a).

Pocas anotaciones después, vuelve sobre el mismo tema:

+ Pr: ¿en qué consiste la identidad de la Persona? no en la concien- 
cia actual, pues entonces no soy la misma persona que era hoy [hace] doce meses, sino mientras pienso en lo que hice entonces. No en la potencial pues entonces todas las personas podrían ser la misma hasta donde sabemos ( $P C 200$ ).

El problema que parece preocupar a Berkeley en estos pasajes podría formularse asi: “¿Qué es lo que hacc que una serie de pensamientos que pueden no ser sucesivos ni continuos unifiquen y sean de una sola persona?" Conforme a la tradición cartesiana que ve el alma como una sustancia pensante, Berkeley encuentra la siguiente dificultad: en caso de que el alma deje de pensar en un momento $a$ y luego reanude su pensar en un momento $b$, posterior al primero, ¿qué sucede con ella en el intervalo $a b$ ? $\_$Podremos, en verdad, afirmar que el alma que dejó de pensar en a es la misma que inicia su pensar en b? ¿Cómo aseguramos la identidad de esta(s) sustancia(s)?

La inclinación de Berkeley, hasta el momento, es la de pensar que hay algo que unifica un conjunto de pensamientos, como pensamientos de una persona. Sin embargo, el elemento unificador no puede ser material o alguna idea:

${ }^{1} \mathrm{M}$ Todas las cosas concebibles por nosotros son $1^{\circ}$ pensamiento, $2^{\circ}$ potencias para recibir pensamientos, $3^{\circ}$ potencias para causar pensamientos ninguna de las cuales es posible que exista en una cosa inerte, insensible ( $P C$ 228).

Si esta observación se liga con la ya citada, $P C 230$-en supra, p. 11—, vemos que Berkeley abandona ahora la tesis de que el alma sea una idea, ya que las ideas son "inertes, sin pensamiento".

La idea sustancialista aún está presente en $P C$ 286:

+ Los pensamientos significan con mayor propiedad, o en su mayor parte se toman como las operaciones interiores de la mente, en donde la mente es activa, aquellos que no obedecen los actos de Volición y en los que la mente es pasiva se denominan con mayor propiedad sensaciones o percepciones. Pero es todo un caso.

En PC 478 comienza a perfilarse una doctrina positiva más clara:

S Pr: ¿cómo se distingue el alma de sus ideas? ciertamente si no hubiese ideas sensibles no podría haber alma, ninguna percepción, remembranza, amor, temor etc. ninguna facultad podría ejercerse.

El comentario inmediato ofrece una respuesta: 
S El alma es la voluntad hablando con propiedad y como tal es distinta de las Ideas (PC 478a).

La propuesta que aquí presenta Berkeley tiene dos aspectos: en $P C$ 478 comienza a perfilarse la tesis humana (cfr. infra, p. ... donde figura la cita de Hume), conforme a la cual si no hay ideas (perceptos, etc.) no hay alma, y en el comentario - PC 478a - aparece un esbozo de la tesis que posteriormente Berkeley desarrollará y verá la luz pública. En este último pasaje, señala una distinción entre alma e ideas e identifica la primera con la voluntad. Sin embargo, antes de decidirse a aceptar claramente esta propuesta, aún considerará otras más con algún detalle. Todavía siguiendo la línea marcada por la última cita, nos da una precisión mayor acerca del alma:

S Pr: ¿no sería mejor no llamar ideas a las operaciones de la mente, confinando aquel término a las cosas sensibles? (PC 490).

Más adelante surge una pregunta capital para la tesis semántica ideista que, hasta ahora, es la que Berkeley ha manejado:

+ Pr: ¿Cómo puede decirse que todas las palabras representan ideas? La palabra Azul representa un Color sin ninguna extensión o abstraído de la extensión. Pero no tenemos una idea de Color sin extensión. no podemos imaginar Color sin extensión (PC 494).

Notemos la presencia en esta cita del signo "+", que señala un rechazo posterior de Berkeley de la propuesta que ahí presenta; sin embargo, lo que aquí me parece interesante señalar es que ahora le brota la inquietud acerca de su propuesta semántica y esto tendrá una importancia capital más adelante.

Algunas notas después surge una observación más pertinente para la caracterización de la sustancia espiritual o mente:

$S$ ¿Qué significa Causa en tanto que diferente de Ocasión? nada sino un Ser que actúa volitivamente (wills) cuando el efecto sigue a la Volición. De esas cosas que suceden desde el exterior no somos nosotros la Causa por tanto hay alguna otra Causa de ellas esto es hay un ser que pone volitivamente (wills) estas percepciones en nosotros ( $P C$ 499).

Pero de inmediato añade un comentario a la nota anterior:

$S$ no ha de decirse nada sino una Voluntad, por ser ininteligible un ser que actúa volitivamente ( $P C 499$ a). 
Conforme a este último comentario parece que Berkeley despoja a la voluntad de un sustrato que realice las voliciones. Una voluntad pura, sin algo que sea lo que tenga tal voluntad. En este punto comienza a surgir la propuesta humeana.

Berkeley continúa precisando más su caracterización de "persona", separándola de las ideas:

S Parece impropio y que conduce a dificultades hacer que la Palabra Persona represente una Idea, o hacer de nosotros Ideas o ideas de las cosas pensantes ( $P C$ 523).

Si aún nos encontramos oficialmente en la posición semántica ideista, Berkeley va a tener cada vez más problemas para conciliar esta posición con el rechazo explícito que hace de la identificación entre ideas y personas. Así, más adelante surge la siguiente observación que pone en jaque la tesis semántica ideista, retomando el tema expresado ya en PC 223 (cfr. supra, p. 11), aun cuando ahora su problema no lo formula en términos de carencia de lenguaje, sino de carencia de ideas:

S Creemos que no conocemos el Alma porque no tenemos ninguna Idea sensible o imaginable anexa a ese sonido. Esto el efecto del prejuicio (PC 576).

Y su comentario inmediato a la nota anterior es:

S Ciertamente no la conocemos, esto está claro si examinamos lo que queremos decir con la palabra conocimiento. $\mathrm{Ni}$ esto arguye defecto alguno en nuestro conocimiento así como tampoco el que no conozcamos una contradicción ( $P C$ 576a).

La tesis sigue en pie, pues sosteniendo la tesis de conocimiento idcista, no podemos conocer lo que no es idea, pues esto sería contradictorio. Sin embargo, Berkeley debería haber apuntado que ni siquiera podemos hablar de ella. Pero, una vez que ha dicho lo anterior, Berkeley señala, en las notas siguientes, algo que lo contradice:

+ La existencia misma de Ideas constituye el alma ( $P C$ 577).

$S$ La conciencia, la percepción, la existencia de Ideas todas parecen ser una ( $P C$ 578).

+ Consulta, arrasa tu Entendimiento qué encuentras ahí además de varias percepciones o pensamientos. Qué quieres decir con la palabra mente debes querer decir algo que percibes o que no percibes, 
una cosa no percibida es una contradicción, querer decir (también) una cosa que no percibes es una contradicción. En esta cuestión las palabras abusan extrañamente de nosotros ( $P C$ 579).

+ La mente es un cúmulo (congeries) de Percepciones. Quita las Percepciones y quitas la Mente pon las Percepciones y pones la mente (PC 580).

+ Dices la Mente no es las Percepciones. sino esa cosa que percibe, Te respondo las palabras esa y cosa abusan de ti éstas son palabras vagas vacías $+\sin$ un significado ( $P C 581$ ).

Aquí tenemos la tesis de la mente como cúmulo (congeries) de percepciones o la tesis humeana del manojo o haz (bundle) de ideas. Presento de inmediato la propuesta de Hume para que el lector pueda constatar la similitud no tan sólo doctrinal sino, además, en la formulación misma de la tesis (véase, asimismo, PC 478 en supra, p. 14):

... cuando penetro de manera más intima en lo que denomino mi-mismo (myself), siempre tropiezo con alguna percepción particular de calor o frío, de luz o sombra, amor u odio, dolor o placer. Nunca, en ningún momento, puedo pescar [me] (catch) a mi-mismo sin una percepción y nunca puedo observar alguna cosa más que la percepción. Cuando se quitan mis percepciones por algún tiempo, como en el sueño profundo, durante el mismo no soy sensible de mi-mismo y en verdad puede decirse que no existe. $\mathrm{Y}$ si todas mis percepciones las eliminase la muerte y yo no pudiese pensar, ni sentir, ver, amar u odiar, tras la disolución de mi cuerpo yo estaría totalmente aniquilado, y no concibo qué más se requeriría para hacerme una perfecta no-entidad (Treat. of $H$. N. I, IV, vi).

Ahora, volviendo a Berkeley, él debe mantener esta tesis si quiere defender, consistentemente, su tesis semántica (y epistémica) ideista. El resultado al que lo lleva una tesis asi es negar que haya algo más, además de ideas, que constituyan una mente, en caso de que también quiera sostener que la mente es cognoscible. Sostener cualquier otra tesis llevaría a Berkeley a una contradicción y él quiere tener cuidado de no cometer el mismo error que le achaca a Locke, de poner, en la base de su caracterización de la mente, un no sé qué como sustrato de las ideas. Con respecto a la voluntad mantiene igualmente que (recuérdese lo ya dicho en PC 499a; supra, p. 15):

* La Voluntad no es distinta de voliciones Particulares (PC 615) y aunque el comentario de Berkeley a esta nota es: 
- Esto alterado de ahora en adelante (PC 615a)

éste debió añadirlo algún tiempo después de registrar la nota principal, pues la tesis aún la mantiene sin alteración avanzadas las notas 600 y no señala cambios radicales hasta $P C 848$.

Por ejemplo, aún en $P C 637$ y 638 leemos, respectivamente:

+ Dices debe haber una sustancia pensante. Algo desconocido que percibe, soporta y liga las Ideas entre sí. Digo, muestra que hay alguna necesidad de ella y por mi la tendrás. No me interesa quitar nada en lo que pueda ver la más mínima razón para creer que deba existir.

+ Afirmo es manifiestamente absurdo, ninguna excusa en el mundo puede darse por qué un hombre haya de usar una palabra sin una idea. Ciertamente encontraremos que cualquier palabra de la que hagamos uso en asuntos de razonamiento puro tiene o debe tener una Idea anexa a ella, esto es: su significado o el sentido en que la tomamos debe ser completamente conocido.

Por último, la siguiente afirmación, $P C 639$, también tiene algo que decirnos en este sentido:

+ Es demostrable: un Hombre nunca puede hacerse que Imagine haya de existir cosa alguna de la que no tiene idea alguna. Quienquiera diga que lo hace, se engaña a sí mismo con Palabras.

Pero cuatro anotaciones después, leemos:

S La gran Causa de perplejidad y oscuridad al tratar la Voluntad, es que Imaginamos que es un objeto de pensamiento (para hablar con el vulgo), creemos que podemos percibirla, contemplarla y verla como cualquiera de nuestras Ideas cuando en verdad no es idea. $\mathrm{Ni}$ hay ninguna Idea de ella. es toto ccelo diferente del Entendimiento esto es de todas nuestras Ideas. Si dices que la voluntad o más bien una Volición es alguna cosa respondo hay una Homonimia en la palabra cosa cuando se aplica a Ideas y voliciones y entendimiento y voluntad. todas las ideas son pasivas, las voliciones activas ... $+(P C$ 643).

Y, al fin, los esperados cambios en la tesis semántica:

S Hay algunas palabras que no representan Ideas, por ejemplo, partículas Voluntad etcétera (PC 661). 
$S$ las partículas representan voliciones y sus Ideas concomitantes (PC 661a).

Una mayor precisión la introduce más adelante:

$S$ Se concede que las Partículas no representan Ideas y sin embargo no se dice que sean sonidos vacíos inútiles. $L a$ verdad acerca de esto es que representan las operaciones de la mente esto es voliciones (PC 667).

Es interesante señalar que en estas tres últimas anotaciones, Berkeley nuevamente recuerda y recoge otra propusta lockeana: si bien es verdad que Locke llegó expresamente a afirmar que

El uso, entonces, de las palabras es el de ser las marcas sensibles de las ideas y las ideas que representan son su significación propia e inmediata (Essay III, ii, 1),

mantiene restringida esta doctrina a los términos generales y más adelante, en el mismo Ensayo, dedica un breve capítulo (I, vii) a la consideración de las partículas (preposiciones, conjunciones, etc.), a las que señala la función "de significar la conexión que la mente confiere a las ideas o a las proposiciones entre sí (III, vii, 1). Berkeley toma la propuesta de Locke en el sentido de que hay expresiones cuya significatividad no depende de que tengan una relación directa con ideas, y la extiende a otras expresiones ("voluntad", por ejemplo, según lo señala en $P C$ 661) que, conforme él lo señala, no aluden a entidades representables mediante ideas. Una formulación paradigmática de su posición figura en $P C$ 782:

G Absurdo Argumentar la Existencia de Dios a partir de su Idea. no tenemos ninguna Idea de Dios. jes imposible!

3. Recapitulación y conclusión. Los pasajes de los $P C$ berkeleyanos que hasta aquí he presentado nos ofrecen una clara visión a) de los problemas a los que Berkeley se enfrentó antes de poder formular una tesis de sustancia espiritual que le satisfaciera, así como $b$ ) de las dudas, cavilaciones y propuestas tentativas de su autor.

Los temas que primeramente influyeron en la configuración del pensamiento de Berkeley, según lo he señalado, fueron i) una tesis semántica ideista estricta, que llegó a tener un gran peso en sus propuestas epistemológicas y ontológicas y ii) un fuerte deseo de evitar los problemas epistémicos que surgen en la filosofía de Locke con respecto a sus 
tesis sobre la sustancia. Con estos dos elementos a la mano, el único resultado posible parecería ser adoptar una posición de tipo humeano con respecto a la sustancia espiritual: no hay alma o espíritu, sino sólo cúmulos de ideas, ya que éstas son los únicos objetos posibles de conocimiento.

Un tema que hizo su aparición en mi escrito al que, sin embargo, Berkeley no parecía prestarle demasiada atención, es el relacionado con la identidad personal. Mi impresión (que espero fundamentar más adelante) es que los problemas relacionados con este asunto son, justamente, los que mueven a Berkeley a adoptar una posición sustancialista con respecto a la mente (espíritu), pues parece que, sin un sustrato, no puede garantizarse que un cúmulo de ideas sea de un sujeto determinado. Pero, para dar el paso adecuado en este sentido, a saber, introducir el elemento sustancial sin que éste origine el problema epistémico de Locke, Berkeley tiene que modificar, primeramente, su tesis semántica y hacerlo de tal manera que sean admisible, como significativos, términos que no tengan un correlato de ideas. La sugerencia para resolver su problema, Berkeley la obtiene de Locke mismo en el tratamiento que este último autor les confiere a las partículas (los términos sincategoremáticos de la tradición) y, una vez que Berkeley tiene en sus manos esta nueva propuesta semántica, puede resolver, entre otros, un grave problema teológico-epistemológico, a saber, "Dios" tiene significado, aun cuando no sea posible tener una idea de Dios.

Una última observación que deseo hacer es que Berkeley puede, según creo, justificar de manera clara la asimetría en su tratamiento de las dos sustancias, la material y la espiritual, señalando que si bien de la espiritual tiene sentido afirmar que no es representable mediante ideas (por ser el sustrato activo de la percepción), esto no es así con respecto al sustrato material lockeano, que evade cualquier posibilidad de ser así representado, aun cuando dada su naturaleza debería ser así representable.

\section{BIBLIOGRAFIA}

[1] Berkeley, G.: Philosophical Commentaries, generally called the Commonplace Book. An editio diplomatica transcribed and edited with introduction and notes by A. A. Luce. Thomas Nelson and Sons Limited. London, 1944.

[2] Hume, D.: $A$ Treatise of Human Nature.

[3] Locke, J.: Essay Concerning the Principles of Human Understanding.

[4] Pitcher, G.: Berkeley (1977). Traducción al español de José A. Robles García. Fondo de Cultura Económica. México, 1983. 